\title{
PERIVASCULAR DELIVERY OF A NITRIC OXIDE DONOR INHIBITS NEOINTIMAL HYPERPLASIA IN VEIN GRAFTS IMPLANTED IN THE ARTERIAL CIRCULATION
}

Aurelio Chaux, $\mathrm{MD}^{\mathrm{a}}$

Xin Min Ruan, MD

Michael C. Fishbein, MD $^{\mathrm{b}}$

Yi Ouyang, $\mathrm{MD}^{\mathrm{a}}$

Sanjay Kaul, $\mathrm{MD}^{\mathrm{c}}$

Jennifer A. Pass, LAT ${ }^{\mathrm{a}}$

Jack M. Matloff, MD
Objective: Nitric oxide has been reported to reduce intimal hyperplasia as a response to arterial injury. This study was designed to assess the possible effect of perivascular application of a nitric oxide donor on neointimal proliferation occurring in veins exposed to the dynamics of the arterial circulation in a hypercholesterolemic rabbit model. Methods: Autologous jugular vein grafts were implanted in the carotid circulation of 20 hypercholesterolemic rabbits. A mixture of a biodegradable polymer and the nitric oxide donor, spermine/nitric oxide, which releases nitric oxide with a half-life of 39 minutes, was applied periadventitially at the time of implantation. Controls were veins bathed in saline solution, polymer alone, and polymer plus the carrier vehicle spermine without nitric oxide. Animals ( $n=5$ in each group) were put to death on day 28 for morphometric analysis, cell count, and immunohistochemical staining. Results: Treatment with perivascular nitric oxide donor significantly decreased wall thickness $(126 \pm 24 \mu \mathrm{m}$ vs $208 \pm 45 \mu \mathrm{m}, p=0.0017)$ and area $\left(124 \pm 22 \mu \mathrm{m}^{2} / \mu \mathrm{m}\right.$ vs $\left.211 \pm 37 \mu \mathrm{m}^{2} / \mu \mathrm{m}, p=0.005\right)$. With the carrier vehicle spermine alone, there was a trend toward reduced intimal thickness, but the change was not statistically significant. In the grafts treated with nitric oxide donor, expression of insulin-like growth factor, fibroblast growth factor, thrombospondins, fibronectin, and tenascin was reduced. Conclusion: The periadventitial delivery of nitric oxide donor produces a reduction of neointimal hyperplasia in veins implanted in the arterial circulation. The mechanism of action is not entirely clear, but the reduction cannot be explained on the basis of decreased cell proliferation alone. Other possibilities are modulation of protein synthesis of vascular smooth muscle cells and production of extracellular matrix components. (J Thorac Cardiovasc Surg 1998;115:604-14)
$V^{\text {en }}$ ein grafts continue to be commonly used as bypass conduits for occluded arteries. Initial patency rates are in the $80 \%$ to $90 \%$ range during the first year but decline to around $50 \%$ at 10 years. ${ }^{1}$

From the Departments of Cardiothoracic Surgery a and Patholo$\mathrm{gy}^{\mathrm{b}}$ and the Division of Cardiology, ${ }^{\mathrm{c}}$ Cedars-Sinai Medical Center, Los Angeles, Calif.

Supported in part by the Western Cardiac Foundation, the Deutsch Family Foundation, and Norman and Georgette Bloom.

Read at the Twenty-third Annual Meeting of The Western Thoracic Surgical Association, Napa, Calif., June 25-28, 1997.

Received for publication July 8, 1997; revisions requested August 18, 1997; revisions received Sept. 18, 1997; accepted for publication Sept. 18, 1997.

Address for reprints: Aurelio Chaux, MD, 10965 Savona Rd., Los Angeles, CA 90077.

Copyright (C) 1998 by Mosby, Inc.

$0022-5223 / 98 \$ 5.00+0 \quad \mathbf{1 2 / 6 / 8 6 3 3 9}$
This decline is apparently related to injury that occurs as a consequence of the venous exposure to arterial hemodynamic conditions, including overdistention, higher intraluminal pressure, and increased sheer stress forces associated with elevated flow velocities. Changes resulting from vascular injury and factors that could lead first to intimal proliferation and then to arteriosclerosis have been extensively studied in arterial as well as venoarterial animal models. ${ }^{2-6}$ Methods to moderate these changes, including the role of nitric oxide (NO), previously referred to as endothelium-derived relaxing factor, in modulating these changes, have been much more extensively studied in arteries than in veins. $^{7,8}$ In these arterial models, NO released luminally by endothelial cells decreases platelet adhesiveness and aggregation. ${ }^{9}$ NO has also been shown to be an important modulator of neutrophil interaction with the endothelium ${ }^{10}$ and of vascular 
smooth muscle cell mitogenesis and proliferation. ${ }^{11}$ The effects of NO on smooth muscle protein synthesis and modulation of extracellular matrix are less well known.

The present investigation was designed to study the less well-defined effects of NO delivered to the perivascular tissues of veins exposed to the dynamics of the arterial circulation. To meet this goal, we achieved perivascular delivery of NO by mixing the $\mathrm{NO}$ donor, spermine/NO that releases NO with a half-life of 39 minutes, with a biodegradable polymer (Atrigel copolymer of polylactic and polyglycolic acid, Comedicus, Inc., Columbia Heights, Minn.). The polymer was applied perivascularly around the vein at the time of implantation into the arterial carotid circulation of New Zealand White rabbits. The effects of perivascular delivery of NO donor on the development of neointima, cell proliferation, and the expression of growth factors and extracellular matrix proteins in venoarterial grafts were examined. Suppression of neointimal hyperplasia by NO donors could potentially improve the patency and prolong the useful life of venoarterial grafts.

\section{Methods}

Animal model. Autologous jugular vein grafts were implanted in the common carotid artery of 20 hypercholesterolemic New Zealand White rabbits weighing between 3 and $3.5 \mathrm{~kg}$. All animals were anesthetized with ketamine $(4 \mathrm{mg} / \mathrm{kg})$ and xylazine $(2 \mathrm{mg} / \mathrm{kg})$, which was mixed and given as a bolus intravenous injection. Anesthesia was maintained with an intravenous solution containing $200 \mathrm{mg}$ of ketamine and $40 \mathrm{mg}$ of xylazine in 250 $\mathrm{ml}$ of normal saline solution. After systemic heparinization, the external jugular vein was exposed on one side of the neck, removed, and flushed with heparinized saline solution. The carotid artery was exposed, and after control of flow was obtained by clamping proximally and distally, the vein segment was reversed and end-to-side anastomoses were performed with the aid of surgical magnification and continuous 8-0 polypropylene sutures. The carotid artery was tied close to the proximal and distal anastomoses and divided in between to direct all of the carotid flow through the vein graft. The average length of the grafts was $3 \mathrm{~cm}$. Animals were fed $2 \%$ cholesterol diets starting 1 week before the operation and continuing throughout the duration of the experiment.

The animals were divided into four groups of five rabbits each. In the first group, the vein was bathed in saline solution before implantation (saline control). In the second group, the biodegradable polymer was used to cover the vein entirely immediately after implantation (polymer control). In the third group, the vein was covered after implantation with a mixture of the polymer and the carrier spermine (spermine control). In the fourth group, the NO donor (spermine/NO) mixed with the biodegradable polymer was applied all around the implanted vein graft. On postoperative day 28 , animals were anesthetized as described earlier; the grafts were dissected and removed together with segments of carotid artery attached proximally and distally. After being flushed with heparinized saline solution, the distal segment of the artery was tied and the graft was distended with $4 \%$ paraformaldehyde until the diameter was the same as in vivo. The proximal portion of the artery was then tied and the graft was immersed in $4 \%$ paraformaldehyde for 1 hour. The tissue was then removed from the paraformaldehyde, washed with a solution of $20 \%$ sucrose, and kept immersed in the same solution at $4^{\circ} \mathrm{C}$ for 12 to 14 hours. Specimens were then transferred to $10 \%$ paraformaldehyde for 8 hours and into $70 \%$ alcohol before they were embedded in paraffin blocks. Animal care complied with the "Principles of Laboratory Animal Care" formulated by the National Society of Medical Research and the "Guide for the Care and Use of Laboratory Animals" issued by the National Institutes of Health (U.S. Department of Health and Human Services, NIH Publication No. 86-23, revised 1985).

Morphometry. In the rabbit, the normal external jugular vein stained with hematoxylin and eosin has a very fragmented and difficult-to-visualize internal elastic lamina and a very thin wall (Fig. $1, A$ ). It is virtually impossible to separate the intima from the media, which together have a small mass, and thereby obtain accurate measurements of each. Consequently, we measured the "proliferative zone," composed of these two layers, to obtain thickness (micrometers), luminal and neointimal circumference, as well as neointimal area (square micrometers per micrometer). These values were obtained by means of a calibrated, computer-based, image-measuring software system (Optimas, BioScan, Inc., Edmonds, Wash.), as described by us previously. ${ }^{12}$ The same computerized system was used to count cells with a light microscope at $20 \times$ magnification (cells per square millimeter) after nuclear staining with hematoxylin.

Immunohistochemical staining. Paraffin-embedded sections (4 mm in thickness) of vein graft specimens were deparaffinized in xylene, rehydrated in decreasing concentrations of alcohol, washed in water, and then submerged in phosphate-buffered saline solution (PBS, Sigma Chemical Co., St. Louis, Mo.) for 15 minutes. Tissue sections were washed with PBS and digested with $0.4 \%$ trypsin (Sigma) for 15 minutes at room temperature. After being washed with PBS, the sections were blocked by $2 \%$ horse serum (Vector Laboratories, Inc., Burlingame, Calif.) in PBS containing $1 \%$ bovine serum albumin (BSA, Sigma), or $3 \%$ BSA in PBS (thrombospondin only) for 30 minutes at room temperature. The tissue sections were then incubated overnight at $4^{\circ} \mathrm{C}$ with monoclonal antibodies $(10 \mathrm{gm} / \mathrm{ml})$ to insulin-like growth factor I (Upstate Biotechnology, Inc., Lake Placid, N.Y.), fibroblast growth factor I (Upstate Biotechnology, Inc.), thrombospondin (Immunotech, Inc., Westbrook, Maine), fibronectin (Chemicon International, Inc., Temecula, Calif.), and tenascin (Chemicon). The second incubation was in biotinylated antimouse immunoglobulin $\mathrm{G}$ or antirat immunoglobulin $\mathrm{G}$ antibodies (Vector), diluted 1:400 in PBS for 30 minutes at room temperature, and streptavidin 

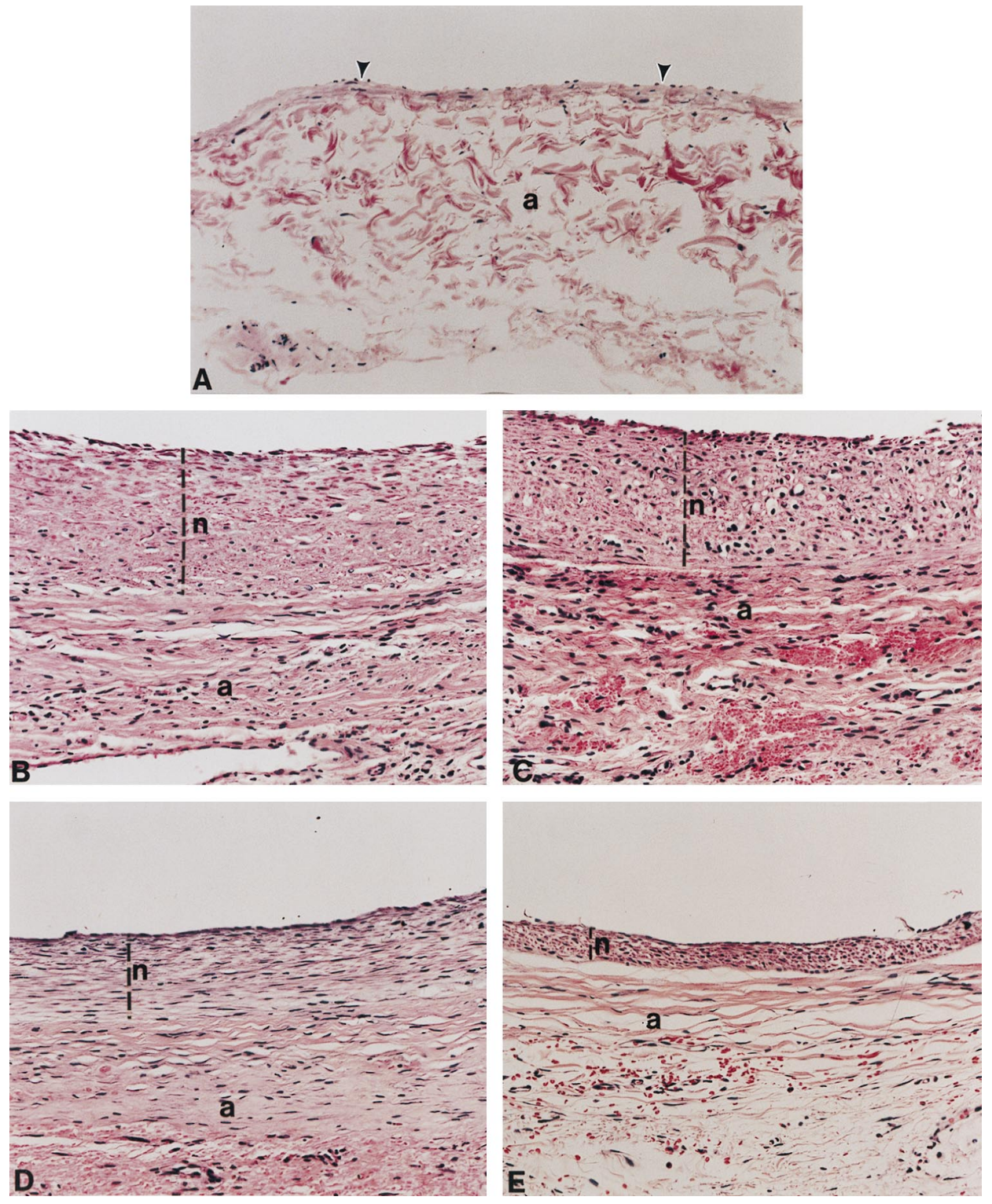

Fig. 1. Sections from normal vein (A), venoarterial graft bathed in saline solution (B), graft covered with polymer only (C), graft covered with polymer + spermine (D), and graft covered with polymer + spermine + NO (E). $a$, Adventitia; $n$, neointima media and intima, or proliferative zone. Arrowheads, Epithelial cells.

conjugated with alkaline phosphatase in PBS (DAKO Corp., Carpinteria, Calif.) for 30 minutes at room temperature. Signal was developed by means of a fuchsin substrate system (DAKO). The sections were counterstained with dilute hematoxylin. Unrelated antigen-specific antibody (mouse immunoglobulin $\mathrm{G}$, negative control from DAKO Corporation) and normal rat serum were used for primary antibody control.

The different intensities of the stain were graded independently by two of the authors (M.C.F. and Y.O). The neoin- tima and the adventitia were graded separately by means of a semiquantitative scale from 0 (negative stain) to 3 (diffuse, intense stain); a grade of 1 was given to focal, mild staining and a grade of 2 to multifocal, moderate staining.

Statistical analysis. Data are presented as mean \pm standard deviation. The analysis of variance using the Bonferroni multiple comparison was performed to determine which two groups were different from each other; the adjusted $p$ value for the required six comparisons should be $0.008(\alpha=0.05 / 6)$. 

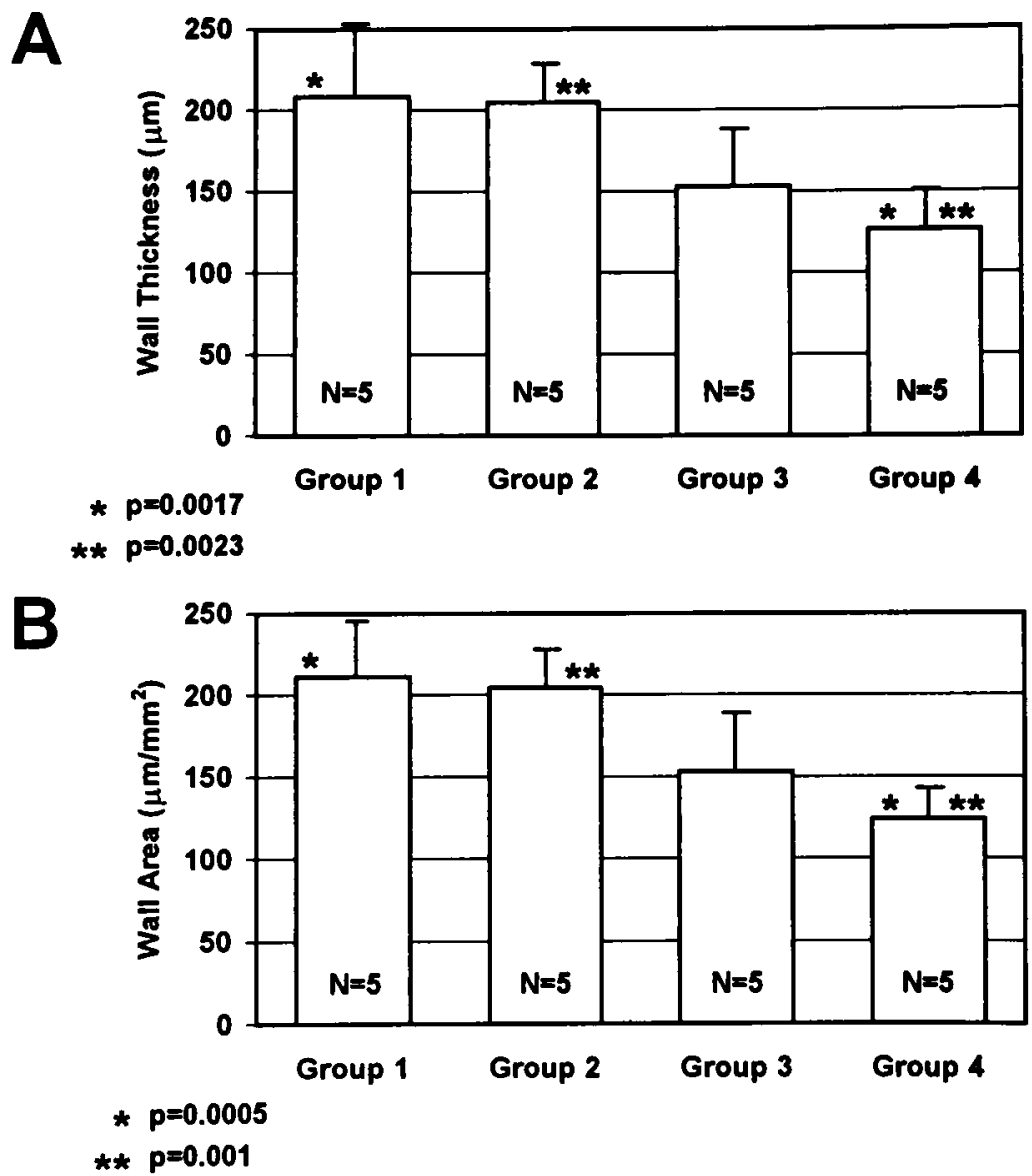

Fig. 2. A, Bar graphs showing the effects of the NO donor (group 4), spermine/NO (group 3), on mean cross-sectional neointimal thickness. B, Mean cross-sectional neointimal area, as compared with saline control (group 1) and polymer alone (group 2).

\section{Results}

The $2 \%$ cholesterol diet was well tolerated. With this concentration of cholesterol in the diet, serum levels rise rapidly and are maintained at values of more than $1000 \mathrm{mg} / \mathrm{dl}$.

All venoarterial grafts in the different groups of animals remained open when the animals were killed, and all had some degree of intimal hyperplasia. Perivascular delivery of spermine/NO using a mixture of a biodegradable polymer resulted in a significant decrease in neointimal thickness and area compared with the saline control group (126 \pm 24 $\mu \mathrm{m}$ vs $208 \pm 45 \mu \mathrm{m}([p=0.0017]$ for thickness and $124 \pm 22 \mu \mathrm{m}^{2} / \mu \mathrm{m}$ vs $211 \pm 37 \mu \mathrm{m}^{2} / \mu \mathrm{m}[p=0.005]$ for area) or the polymer control group (126 \pm 24 $\mu \mathrm{m}$ vs $205 \pm 23 \mu \mathrm{m}[p=0.0023]$ for thickness and $124 \pm 22 \mu \mathrm{m}^{2} / \mu \mathrm{m}$ vs $204 \pm 27 \mu \mathrm{m}^{2} / \mu \mathrm{m}[p=0.0011]$ for area) (Fig. 2). With the polymer mixed with the carrier spermine alone, there was a trend toward a reduction in neointimal formation, but the effect did not achieve statistical significance when compared with saline or polymer only controls. The inhibitory effects of polymer + spermine were not significantly different from those caused by polymer + spermine/NO (Fig. 2). Microscopically, the grafts continued to have a single layer of endothelial cells, beneath which were layers of smooth muscle cells interspaced with intercellular matrix (Fig. 1). There was a trend toward a reduction in the number of cells per square millimeter in NO-treated animals (3179 \pm 799$)$ as compared with the saline $(4542 \pm 614)$, polymer $(4193 \pm 986)$, and spermine $(4057 \pm 754)$ controls, although the differences were not enough to achieve significance $(p=$ 0.08 ) when tested by means of the Bonferroni modification for multiple comparisons (Fig. 3). We believe that the decrease in cell numbers is not enough to account for the total differences in thickness and area among all groups; consequently, the reduction of ex- 


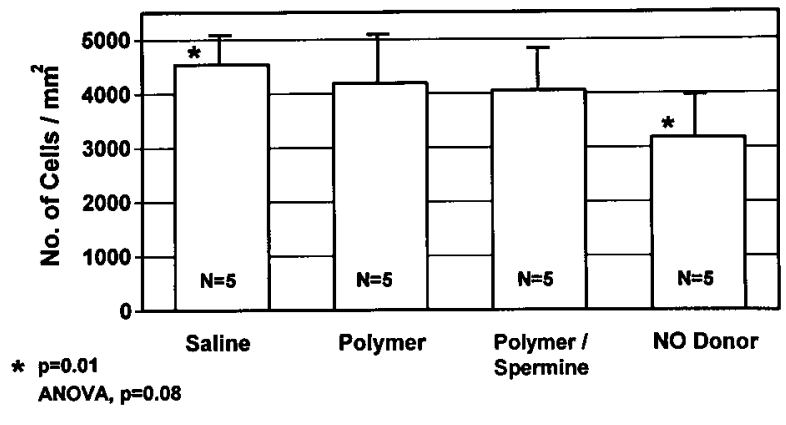

Fig. 3. Effect of NO donor on cell density, compared with saline, polymer, and polymer+spermine controls. ANOVA, Analysis of variance.

tracellular matrix becomes an important factor in explaining this phenomenon. The extracellular matrix components studied by immunohistochemical staining were reduced in the NO donor-treated grafts; this reduction was more significant for thrombospondins $(1 \pm 0.5$ vs $3 \pm 0.5)$ (Fig. 4) and less so for fibronectin $(1 \pm 0.5$ vs $2 \pm 0)$ and tenascin-A $(1 \pm 0.2$ vs $2 \pm 0.2)$. Of the two growth factors studied, expression of insulin-like growth factor I was significantly reduced by NO donor administration (0.6 vs $2 \pm 0.4$ ) (Fig. 5); a slightly less significant reduction was noted for fibroblast growth factor I $(2 \pm 0$ vs $3 \pm 0)$.

The polymer application resulted in some degree of inflammatory reaction of the surrounding tissues in two thirds of the animals in which it was applied; two had clear abscess formation necessitating drainage. Despite these findings, the NO donor application demonstrated an effect, and all grafts remained open in this series of experiments.

\section{Discussion}

Neointima is, by definition, a new and usually thickened layer that forms in response to vascular injury. In the rabbit jugular vein it is possible to recognize a new proliferative zone because the normal vein wall has a single layer of endothelial cells, a very thin layer of intima and media formed by a few layers of smooth muscle cells, an almost nonexistent internal elastic lamina, and a loose but abundant layer of adventitial tissue that contains scattered cells (Fig. 1, $A$ ).

New intimal formation has been described as an important stage in the response to vascular injury, as well as a precursor to stenosis and formation of arteriosclerotic lesions. ${ }^{6}$

Several mechanisms have been postulated to explain the development of intimal hyperplasia, among them, the influence of hemodynamic factors such as turbulence, wall shear stresses and compliance mismatch, interactions between components of the vessel wall (endothelial cells, smooth muscle cells), and elements of the blood (e.g., platelets, macrophages, and leukocytes). In all of these hypothesized mechanisms, the central theme has been the initial occurrence of endothelial injury. ${ }^{7}$

The best known mitogen for smooth muscle cells is basic fibroblast growth factor, and the best known migration stimulator is platelet-derived growth factor. ${ }^{6,7}$ Our attempts to study expression of plateletderived growth factor in this model were not successful because at the time the experiments were carried out, the only primary antibody available was an antihuman rabbit-produced antibody; the secondary antibody for immunostaining has to be antirabbit, and in this model it produces a very significant background staining, making the interpretation of the results difficult and unreliable. Little is known about other molecules controlling the response of vein conduits when they are exposed to arterial hemodynamics in human beings or in other complex animal species, thus our interest to study insulin-like growth factor I and several matrix proteins expression.

Several agents have been tested experimentally and clinically to reduce neointimal formation in injured arteries, as well as in vein grafts. Some of these methods have been successful in the animal model, but when applied clinically, the results have been inconsistent at best, and commonly ineffective; such is the case of the platelet inhibitors aspirin and dipyridamole, ${ }^{13,14}$ as well as heparin ${ }^{15}$ steroids, ${ }^{16}$ and photodynamic therapy of vein grafts. ${ }^{17}$ In an attempt to achieve a more efficient control of neointimal growth, different approaches have recently been taken, such as gene therapy with the use of an adenovirus vector ${ }^{18}$ or antisense oligonucleotide blockage of medial smooth muscle cell proliferation. ${ }^{19}$ Much attention has been given to the study of NO in the prevention of neointimal formation, given its known effects on vasomotor tone, ${ }^{20}$ platelets ${ }^{21}$ neutrophil interaction with the endothelium, ${ }^{10}$ and smooth muscle cell proliferation. ${ }^{11}$ Less is known regarding the effect of NO on smooth muscle cell protein synthesis, including production of collagen by these cells.

The methods used to increase the presence of NO in the arterial wall have ranged from supplementation with the nitric oxide precursor L-arginine, ${ }^{22}$ NO-generating compounds such as S-nitroso- $N$ acetylpenicillamine, and sodium nitroprusside,$^{23}$ to local delivery of NO-releasing donor compounds. ${ }^{24}$ 

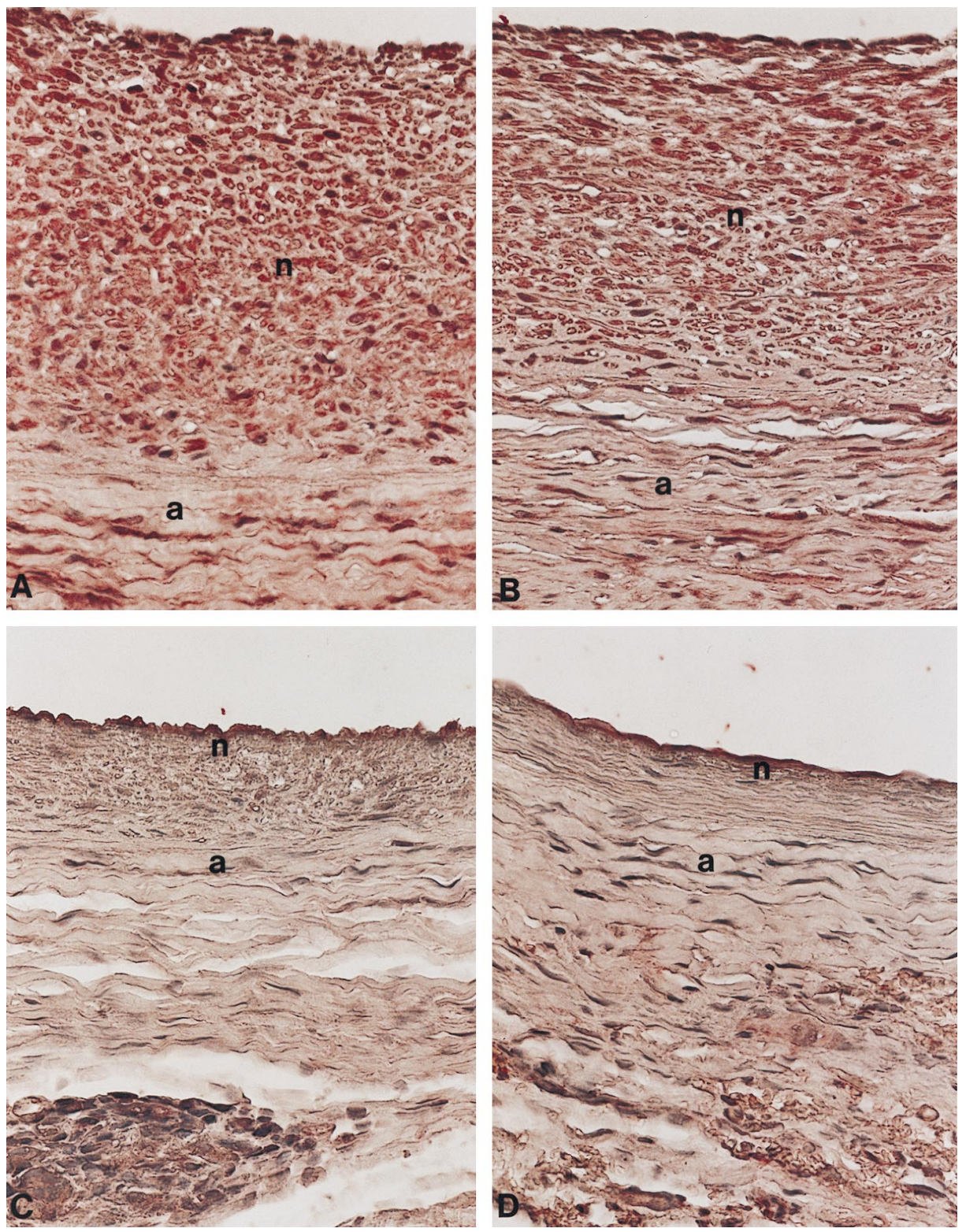

Fig. 4. Immunohistochemical studies of expression of thrombospondins in venoarterial grafts. Group 1, saline (A); group 2, polymer (B); group 3, polymer+spermine (C); group 4, polymer +spermine/NO (D). Note the almost complete absence of thrombospondin expression by cells in the NO-treated graft (D). $n$, Neointima (media and intima); $a$, adventitia. (Original magnification $\times 50$.)

Local delivery may offer the advantage of preserving desired regional effects on platelets, smooth muscle cells, macrophages, and lymphocytes, as well as on extracellular matrix proteins, while systemic effects such as hypotension are minimized. This approach also circumvents the rapid inactivation of NO by hemoglobin in the circulating blood before achieving high concentrations at the target site.

Dietary fat intake and high levels of plasma lipids have been recognized as major risk factors in the development and progression of arteriosclerosis. They influence both platelet and endothelial cell function in human beings and experimental models. ${ }^{25}$ Badimon and colleagues ${ }^{26}$ have shown that high plasma lipid levels may contribute to acute thrombosis through a mechanism by which plateletvessel wall interactions are enhanced and modulated because of changes in platelet reactivity and red 

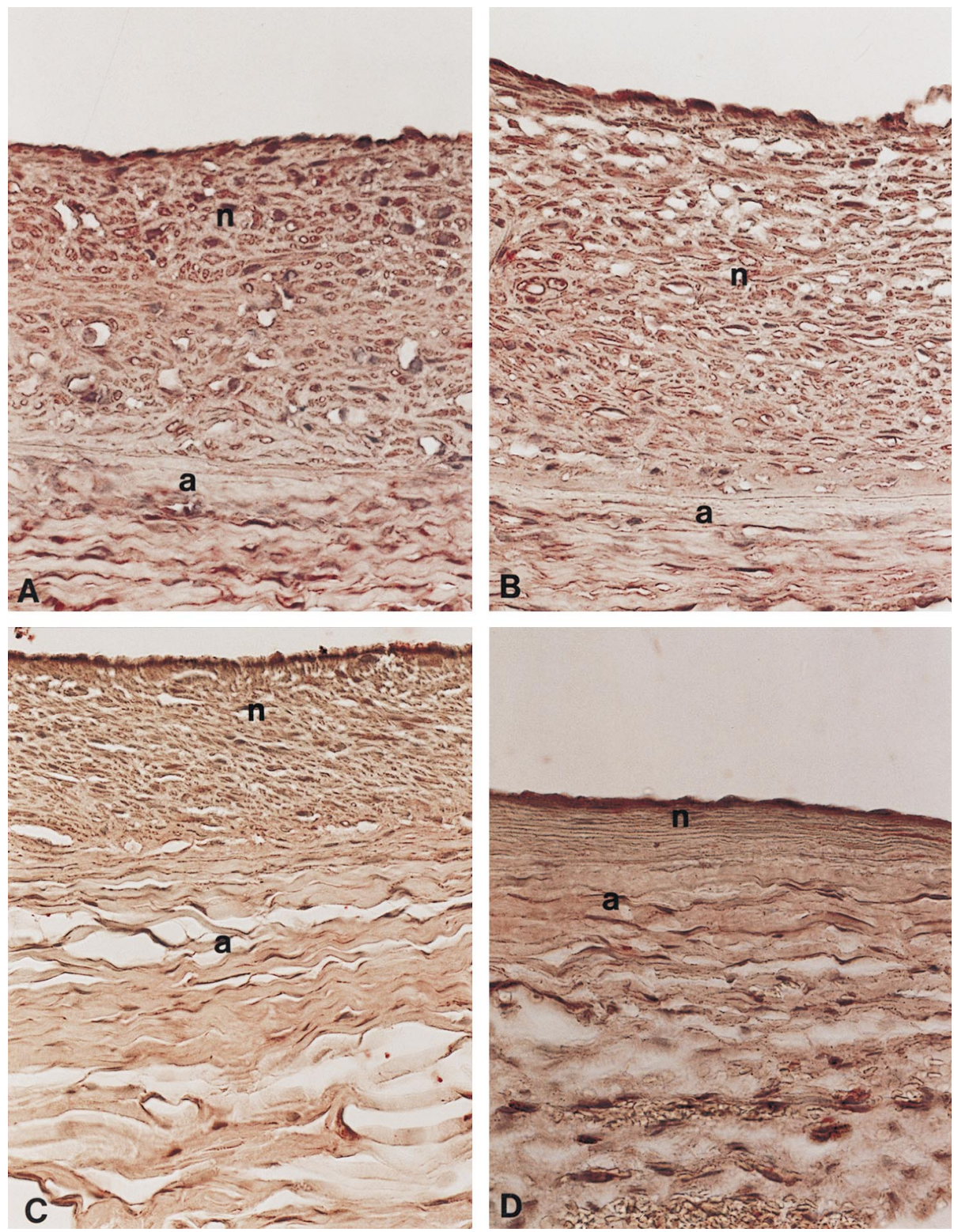

Fig. 5. Expression of insulin-like growth factor in venoarterial grafts. Group 1, Saline (A); group 2, polymer (B); group 3, polymer + spermine $(\mathbf{C})$; group 4, polymer + spermine/NO (D). $n$, Neointima (media and intima); $a$, adventitia. (Original magnification $\times 50$.)

blood cell shape deformation in relation to cholesterol levels.

We have used a hypercholesterolemic rabbit model to demonstrate the importance of the presence of vein valves in the formation of arteriosclerosis-like lesions in venoarterial grafts. ${ }^{12}$ In that study we noted that thickness and area of the neointima are increased at 4 weeks after implantation of the vein graft into the carotid circulation and that cell proliferation peaked at that time as well.
On the basis of this experience, we decided to use a similar model to test the effect of periadventitial local delivery of NO in neointimal proliferation.

The histologic sections show cells surrounded by the extracellular matrix that provide support to the tissue (see Figs. 1 and 4). A major component of extracellular matrix consists of various types of collagen, fibronectin, tenascin, and thrombospondins, among other protein compounds.

Fibronectin is a glycoprotein that couples in high 
proportion with polysaccharides, galactose, and other sugars; it is common in neointima and it binds to other proteins such as collagen and fibrin. Fibronectin is involved in the spreading and locomotion of cells and in the adhesion of fibroblasts to tissue. It is also found in the $\alpha$-granules of platelets, and it may enhance their aggregation and adhesion to mural thrombus at sites of vascular injury. Fibronectin associates strongly with actin, is synthesized by the fibroblast, and thus is an important mediator of neointimal thickening. Tenascin and thrombospondins are large-matrix glycoproteins. Their function may be important in converting quiescent smooth muscle cells to active forms of a different phenotype capable of migration and proliferation. The large size and multi-domain structure of tenascin and thrombospondins suggest the presence of these proteins in the early formation of matrix. ${ }^{27}$ The results obtained in this study indicate that the method of perivascular delivery of an NO donor is effective in achieving a decrease in neointimal formation. The mechanism of this action is not explained by the data obtained in this study; however, the action appears to be due to a combination of NO donor-induced impaired mitogenesis and a reduction of expression of several extracellular matrix proteins such as thrombospondins, fibronectin, and tenascin. These data are consistent with those of other investigators studying vascular smooth muscle cells. $^{23}$

Because of the lack of statistical significance between the effects of the carrier spermine alone and the NO donor (spermine/NO), it is not possible to conclude that NO alone was responsible for the effects seen in the study. Without determination of tissue levels of cyclic guanosine monophosphate (a marker of the effect of NO), as suggested by Park and associates,${ }^{28}$ it is difficult to clarify this issue. We propose that in future investigations tissue levels of cyclic guanosine monophosphate should be measured and that other NO donors that do not include spermine, such as DEA-NO (diethylamino-NO), SNAP (S-nitroso- $N$-acetylpenicillamine), or GSMO (nitroso-glutathione), could be used to compare their effects with those of local perivascular delivery of NO and to help delineate the effect of the carrier.

The inhibitory effect of the carrier spermine suggested in these experiments supports the recent findings of other researchers. ${ }^{29,30}$ The exact mode of action of spermine, however, continues to be a subject of investigation. The importance and the different roles of the extracellular matrix compo- nents to the formation of neointimal hyperplasia have only very recently been considered, and further studies such as this one should increase the possibility to target the process as a method to limit neointimal hyperplasia.

The major limitation of this delivery method for NO in human beings would be due to technical difficulties in controlling the application of the polymer + spermine/NO mixture and the inflammatory reaction that it produces in the surrounding tissues. Other methods of local NO delivery are under investigation.

We thank Meenu Sandhu for statistical analysis of the data and Kathleen Farrington and Michele A. DeRobertis for their assistance in the preparation of this manuscript.

REFERENCES

1. Bourassa MG. Fate of venous grafts: the past, the present and future. J Am Coll Cardiol 1991;17:1081-3.

2. Bjorkerud S, Bondjers G. Arterial repair and atherosclerosis after mechanical injury: tissue response after induction of a large superficial transverse injury. Atherosclerosis 1973;18(Pt 5):235-55.

3. Clowes AW, Schwartz SM. Significance of quiescent smooth muscle migration in the injured rat carotid artery. Circ Res 1985;56:139-45

4. Ross R. The pathogenesis of atherosclerosis: a perspective for the 1990s. Nature 1993;362:801-13.

5. Zwolak RM, Kirkman TR, Clowes AW. Atherosclerosis in rabbit vein grafts. Arteriosclerosis 1989;9:374-9.

6. Schwartz SM, deBlois D, O'Brien ERM. The intima: soil for atherosclerosis and restenosis. Circ Res 1995;77:445-65.

7. Chervu A, Moore WS. An overview of intimal hyperplasia. Surg Gynecol Obstet 1990;171:433-47.

8. Wennmalm A. Nitric oxide (NO) in the cardiovascular system: role in atherosclerosis and hypercholesterolemia. Blood Pressure 1994;3:279-82.

9. Bassenge E. Antiplatelet effects of endothelium-derived relaxing factor and nitric oxide donors. Eur Heart J 1991; 12(Suppl E):12-5.

10. Provost P, Lam JYT, Lacoste L, Merhi Y, Waters D. Endothelium-derived nitric oxide attenuates neutrophil adhesion to endothelium under arterial flow conditions. Arterioscler Thromb 1994;14:331-5.

11. Garg UC, Hassid A. Nitric oxide-generating vasodilators and 8-bromo-cyclic guanosine monophosphate inhibit mitogenesis and proliferation of cultured rat vascular smooth muscle cells. J Clin Invest 1989;83:1774-7.

12. Chaux A, Ruan XM, Fishbein MC, Sandhu M, Matloff JM. Influence of vein valves in the development of arteriosclerosis in venoarterial grafts in the rabbit. J Thorac Cardiovasc Surg 1995;110:1381-90.

13. Radic ZS, O’Malley MK, Mikat EM, Makhoul RG, McCann $\mathrm{RL}$, Cole CW, et al. The role of aspirin and dipyridamole on vascular DNA synthesis and intimal hyperplasia following deendothelialization. J Surg Res 1986;41:84-91.

14. Pantely GA, Goodnight SH, Rahimtoola SH, Harlan BJ, DeMots H, Calvin L, et al. Failure of antiplatelet and 
anticoagulant therapy to improve patency of grafts after coronary artery bypass. N Engl J Med 1979;301:962-6.

15. Clowes AW, Clowes MM. Kinetics of cellular proliferation after arterial injury. IV. Heparin inhibits rat smooth muscle mitogenesis and migration. Circ Res 1986;58:839-45.

16. Chervu A, Moore WS, Quinones-Baldrich WJ, Henderson T. Efficacy of corticosteroids in suppression of intimal hyperplasia. J Vasc Surg 1989;10:129-34.

17. LaMuraglia GM, Klyachkin ML, Adili F, Abbott WM. Photodynamic therapy of vein grafts: suppression of intimal hyperplasia of the vein graft but not the anastomosis. J Vasc Surg 1995;21:882-90.

18. Kupfer JM, Ruan XM, Liu G, Matloff J, Forrester J, Chaux A. High-efficiency gene transfer to autologous rabbit jugular vein grafts using adenovirus-transferrin/polylysine-DNA complexes. Hum Gene Ther 1994;5:1437-43.

19. Mann MJ, Gibbons GH, Kernoff RS, Diet FP, Tsao PS, Cooke JP, et al. Genetic engineering of vein grafts resistant to atherosclerosis. Proc Natl Acad Sci U S A 1995;92:4502-6.

20. Shepherd JT, Katusic ZS. Endothelium-derived vasoactive factors. I. Endothelium-dependent relaxation. Hypertension 1991;18(Suppl III):III76-85.

21. Radomski MW, Palmer RMJ, Moncada S. Endogenous nitric oxide inhibits human platelet adhesion to vascular endothelium. Lancet 1987;2(8567):1057-8.

22. Davies MG, Dalen H, Kim JH, Barber L, Svendsen E, Hagen P-O. Control of accelerated vein graft atheroma with the nitric oxide precursor: 1-arginine. ${ }^{1}$ J Surg Res 1995;59:35-42.

23. Kolpakov V, Gordon D, Kulik TJ. Nitric oxide-generating compounds inhibit total protein and collagen synthesis in cultured vascular smooth muscle cells. Circ Res 1995;70:305-9.

24. Xu X-P, Cercek B, Meisel SR, Dimayuga P, Sharifi B, Ong JM, et al. Nitric oxide donor suppresses vascular smooth muscle cell proliferation via inhibition of nuclear factor kappa-B. Circulation 1996;94:8(Suppl):I1472.

25. Nordoy A. Dietary fatty acids, platelets, endothelial cells and coronary heart disease. Acta Med Scand Suppl 1985;701:1522.

26. Badimon JJ, Badimon L, Turitto VT, Fuster V. Platelet deposition at high shear rates is enhanced by high plasma cholesterol levels: in vivo study in the rabbit model. Arterioscler Thromb 1991;11:395-402.

27. Majesky MW. Neointima formation after acute vascular injury: role of counteradhesive extracellular matrix proteins. Tex Heart Inst J 1994;21:78-85.

28. Park KH, Rubin LE, Gross SS, Levi R. Nitric oxide is a mediator of hypoxic coronary vasodilation. Circ Res 1992;71: 992-1001.

29. Monti MG, Pernecco L, Manfredini R, Frassineti C, Barbieri $\mathrm{D}$, Marverti G, et al. Inhibition of cell growth by accumulated spermine is associated with transient alteration of cell cycle progression. Life Sci 1996;58:2065-72.

30. Bardocz S, Sakhri M, Pusztai A, Maguire NM, Lin PK. Effect of three novel polyamine oxa-analogues (MTR-OSPD, DIPSPN and APPO-TFA) on the growth and proliferation of Swiss 3 T3 cells. Int J Biochem Cell Biol 1996;28:697-704.

\section{Discussion}

Dr. Edward D. Verrier (Seattle, Wash.). Current theories of atherosclerosis all revolve around the "endothelial injury" theory, which is then affected by a host response involving components of the vessel wall and elements within the blood. The responding lesion of early intimal proliferation is characterized by smooth muscle migration to the intima, cellular proliferation, and extracellular matrix elaboration. This sequence has been extensively characterized in a number of animal models including the rabbit jugular vein/carotid artery model used by the authors. NO was the molecule of the year in 1992 and continues to be studied extensively. NO has a number of effects on the vasomotor properties of the endothelial cells, platelet activation, neutrophil interaction, and smooth muscle proliferation. NO should be theoretically favorable in preventing neointimal hyperplasia. The delivery model of NO to the adventitia of the jugular vein using the polyamine vehicle is the model chosen by these authors.

I have a number of questions. As they noted, the major concern of their conclusions is the fact that both spermine and spermine with $\mathrm{NO}$ had an effect, and that spermine had an independent effect. Spermine alone mixed with the biodegradable polymer had an effect, as did the spermine/NO polymer. If the group size had been larger, the spermine polymer would probably have reached statistical significance by comparison with the controls. There were no differences between the spermine polymer and spermine NO/polymers group. To show NO dependence, did you consider other NO donor agents, such as diethylamino NO, or SNAP (S-nitroso- $N$-acetylpenicillamine) NO, or nitrosoglutathione, which are all NO carriers? By using these agents and consistently showing that NO donor agents had a greater effect than the vehicle alone, the argument would be considerably stronger.

Dr. Chaux. You are absolutely correct. We have considered using other NO donors. Unfortunately, our laboratory is in a transition period, and that work will have to be completed in the future. There is an opportunity for groups interested in this subject to take over this work. Yes, there are ways to differentiate if the effects seen in these experiments are indeed due to NO or if they are due to spermine. I believe the effects are probably due to combined effects. There have been recent publications on the effects of spermine as an isolated agent on intimal hyperplasia.

Dr. Verrier. The half-life of spermine is only $39 \mathrm{~min}$ utes. It is hard to believe that this short-term exposure to NO had any effect on the overall development of neointimal hyperplasia. If this is the case, then why wouldn't the infusion of sodium nitroprusside or nitroglycerin have an effect? In fact, they probably do. However, the exposure has to be much longer if not continuous. For years, we have given nitroglycerin and nitroprusside postoperatively to our patients who have had vascular and cardiac operations, and neointimal hyperplasia still develops in many of them. The literature contains a number of studies addressing this issue. The first involved balloon angioplasty on the rat aorta. The rats were then immediately placed in living chambers where the content of air inhaled could be controlled. Rats in the experimental group were chronically exposed to low levels of NO; the control group had normal air. When they were killed, the animals that had been breathing the gas mixture with NO had no neointimal hyperplasia, whereas the control animals did. The investigators then took the study a step further and 
took some of the animals out of the NO environment after some period of time and had them inspire normal air. When the animals were later killed, the rats that had been exposed to only NO still had no intimal hyperplasia, whereas those that had been initially placed in the NO environment and then moved to air did have intimal hyperplasia. Thus the study suggests that long-term exposure to NO is needed to prevent intimal hyperplasia. Early exposure to NO may only delay the development and not prevent the occurrence of intimal hyperplasia. Your thoughts?

Dr. Chaux. This is a very controversial area. The working hypothesis, which has not yet been proved, can be considered as a first step. It is based on the possibility that local delivery is different from systemic delivery of NO. If local delivery can produce very high initial concentrations and, by doing so, can stop the very initial injury changes that occur at the endothelial as well as subendothelial and adventitial layers, perhaps we can modify the rest of the processes that eventually will result in neointimal hyperplasia. At present, this is theoretical, and I do not mean to claim that this indeed will be the case.

Dr. Verrier. The inflammatory reaction of the carrier is of significant concern. In fact, we are postulating that the number of cells decreases with the NO carrier group, and yet the inflammatory response, at least in two thirds of those, is increased. An inflammatory response would be expected to elicit a cellular reaction. How do you rectify those two differences, particularly in the one third of the animals that did not have the inflammatory response? Was that different from those that had inflammation?

Dr. Chaux. Yes, it is surprising that the inflammatory reaction produced by this polymer was more pronounced in the surrounding tissues than in the graft itself. The grafted veins were all patent, even in the presence of an "abscess" surrounding some of them, and it was possible to dissect the grafts easily. A significant amount of inflammatory reaction was noticed in the muscular layers around the graft. We performed cell counts in the grafts four different times and we could not find significant differences. We are merely reporting the observation without a good explanation of why the inflammatory changes noted were mostly in the surrounding tissues and not in the grafts.

Dr. Verrier. How were the cell counts done, and do cell counts measure cellular proliferation? Did you consider using proliferating cell nuclear antigen or any other nuclear assays of proliferation, which are shown to be significantly more accurate than the subjective assessment of counting cells?

Dr. Chaux. We used hematoxylin stain for nuclei. In this series of experiments, we did not use proliferating cell nuclear antigen, as we have done and published in the past. After the nuclei were stained, they were counted by means of the Optimas system and software.

Dr. Verrier. Immunocytochemistry was used to analyze your extracellular matrix protein and growth factors. Quantitating differences can be very difficult because visual assessment of different intensities of stain is subjective. Could you better describe this approach? Were your specimens randomized and were the reviewers blinded to the protocols? How consistent were the observations between observers?

Dr. Chaux. The specimens were not randomized and the reviewers were blinded. One of the reviewers and coauthors was Dr. Michael Fishbein, senior pathologist at Cedars-Sinai. The other one was a technician in our laboratory who also did the staining in a blinded fashion. You are correct in saying that this is a subjective method and that it is difficult to quantify the differences. Pathologists do accept these gradings, and there are plenty of reports in the literature to document grading expressions between 0 and 3. A 0 gradient means that there is no expression at all, grade 1 is a very weak expression, grade 2 is an intermediate expression, and grade 3 corresponds to a strong expression of either growth factors or extracellular matrix proteins.

Dr. Verrier. Why did you elect to study insulin-derived growth factor rather than platelet-derived growth factor? Platelet-derived growth factor is the most well-characterized and strongest smooth muscle mitogen.

Dr. Chaux. Right. Unfortunately, there is no available platelet-derived growth factor secondary antibody that can be used in the rabbit model. The secondary antibodies available for immunostaining are antirabbit, and if used in this model they produce extensive background staining. We searched and searched to see if there were plateletderived growth factor antibodies produced in another animals that could be used in the rabbit model, and we could not find any. We considered changing our model to rodents or to other mammals, but that was not very practical for us. That is the reason we did not study platelet-derived growth factors. In trying to choose another growth factor, we arbitrarily decided on insulinderived growth factor because its expression and effects are less well known and the antibody is readily available for use in this rabbit model.

Dr. Verrier. This observational study is an important step in understanding NO influence on vascular cellular responses. I believe we must be very careful in making any cause-and-effect conclusions. The relationship between endothelial activation, smooth muscle responses, matrix effects and growth factor, cytokines and NO, or other endothelial-derived factors, are very complex; therefore we need to be very careful about the conclusions, particularly those between cause and effect.

Dr. David A. Fullerton (Winnetka, Ill.). I think you are on to something here, for the following reasons. A flurry of publications have appeared recently in the basic science journals looking at the effects of ionized radiation on preventing restenosis after angioplasty, and for that reason there is a push to develop radioactive stents. The surgeon's analogy to that is to be able to provide something topical, as you have done now at the time of surgery. I am compelled that you have used the standard model for generating atherosclerosis. Your control data are histologically identical to those published by other investigators, and I am persuaded that in your treatment groups, you diminished the proliferation in the neointima.

Acknowledging that NO may or may not be the mechanism by which you achieved your results, from a pragmatic standpoint, do you think it is possible to overcome 
the problems you identified in terms of just the cumbersome nature of the polymer? It would seem to me that some engineer ought to be able to design a readily usable polymer that you could just drip onto the anastomosis. Perhaps you do not even need NO in it; perhaps it is just spermine or some other compound. Can those problems be overcome?

Dr. Chaux. Yes, I think that they can. I believe the saphenous vein can be modified to make it a better conduit. To that effect, workers at Stanford are trying to manipulate genes. We have done some of that work as well. We have now completed a different series of experiments in which an external stent is placed around the vein; there is a plan to use low-radiation stents as well.
With respect to NO in particular, I think it is possible to modify the method that we used. For instance, it is possible to place a layer of some kind of different material, with or without the stent, and deliver high concentrations of NO to the adventitia of the vessel. I believe, as do many others, that the adventitia is going to be extremely important in understanding the biology of vein conduits. As Dr. Verrier mentioned before, the initial endothelial injury is important, but there is increasing evidence that the process of response to vessel injury comes from the inside layers of the vessel, as well as the outside layers. It is a fascinating field; there is a lot of room here for young investigators, and for others not so young, who want to have a little fun. 